\title{
Fitness Level is Associated with Sex-Specific Regional Fat Differences in Normal Weight Young Adults
}

\author{
Tyler A. Bosch ${ }^{1 *}$, Donald R. Dengel ${ }^{2,3}$, Justin R. Ryder ${ }^{3,4}$, Aaron S. Kelly ${ }^{1,3}$ and Lisa Chow ${ }^{1}$ \\ ${ }^{1}$ Department of Medicine, University of Minnesota Medical School, Minneapolis, MN 55455, USA \\ ${ }^{2}$ School of Kinesiology, University of Minnesota, Minneapolis, MN 55455, USA \\ ${ }^{3}$ Department of Pediatrics, University of Minnesota Medical School, Minneapolis, MN 55455, USA \\ ${ }^{4}$ Division of Epidemiology \& Community Health, University of Minnesota School of Public Health, Minneapolis, MN 55455, USA
}

Received: March 31, 2015; Accepted: May 04, 2015; Published: May 20, 2015

*Corresponding author: Tyler A. Bosch, Department of Medicine, University of Minnesota Medical School, MMC 101420 Delaware Street SE, Minneapolis, MN 55455, USA, Tel: 612-301-8309; Fax: 1612-626-3133; Email: bosch041@umn.edu

\begin{abstract}
Objectives: To characterize regional body composition and insulin sensitivity differences between young adults who were normal weight with either high or low fitness determined by $\mathrm{VO}_{2}$ peak. We hypothesized that higher fitness levels would be associated with reduced visceral fat (VAT) and improved insulin sensitivity.

Design: A cross-sectional comparison of normal weight males and females with high or low fitness matched on age and sex.

Methods: A total of 38 (20M/18F) individuals were recruited for this study. Thirty-two young adults $(18 \mathrm{M} / 14 \mathrm{~F})$ were matched on age (mean $22.5 \pm 3$ yrs.) and BMI $\left(22.4 \pm 2.4 \mathrm{~kg} / \mathrm{m}^{2}\right)$ and sex and classified by high or low fitness based on $\mathrm{VO}_{2}$ peak difference $(\geq 8 \mathrm{ml} / \mathrm{kg} / \mathrm{min})$. Total and regional body composition, including VAT, was measured by Dual Energy X-Ray Absorptiometry (DXA). Insulin sensitivity was measured by hyperinsulinemic-euglycemic clamp. An analysis of variance compared regional body composition and insulin sensitivity between high and low fitness young adults with a normal BMI.

Results: Higher fitness was associated with significantly lower percent body fat, lower android fat mass and higher insulin sensitivity in males $(-7.2 \%, P<0.001 ;-0.5 \mathrm{~kg}, P=0.048 ; 5.6 \mathrm{mg} / \mathrm{kg}(\mathrm{FFM}) / \mathrm{min}$, $\mathrm{p}=0.002)$. In females, higher fitness was associated with significantly lower percent body fat, lower leg fat but no difference in insulin sensitivity $(-6.7 \%, P=0.001 ;-2.7 \mathrm{~kg}, P<0.001 ; 2.5 \mathrm{mg} / \mathrm{kg}(\mathrm{FFM}) / \mathrm{min}$, $P=0.40$ ). No differences in VAT were observed between high and low fitness groups. Interestingly in females, there was no difference in total lean mass, trunk lean mass or leg lean mass $(P=0.59, P=0.17$, $P=0.99$ ).
\end{abstract}

Conclusion: Higher fitness does not influence VAT in normal weight individuals. Sex influenced regional fat and insulin sensitivity differences between high fitness and low fitness groups.

Keywords: Adipose Tissue; Insulin Resistance; Obesity Paradox; DXA; Body Composition

\section{Introduction}

Body Mass Index (BMI) may misclassify people as "normal weight" because it does not detail the composition of tissue
$[1,2]$. Individuals with higher percent body fat, at a given level of BMI, had a higher prevalence of prediabetes, type 2 diabetes and an adverse cardiovascular profile [1,2]. The differences in percent fat are due to differences in regional fat mass. Two regional depots have become of interest in their relation to sexspecific fat distribution; the android (abdominal) and gynoid (hip/gluteal) regions. Males generally accumulate more fat in the android region. In contrast females generally accumulate more fat in the gynoid region. This distinction is thought to be a factor explaining why females have lower cardiometabolic risk [3-5], prior to the onset of menopause. Visceral Adipose Tissue (VAT) is located deep within the android region in the compartments surrounding the visceral organs. VAT, even beyond total body fat, is an independent marker for cardiometabolic risk [6-8]. While regional fat differences have been measured extensively in older and overweight/obese populations [9-11], very few studies have focused on regional fat differences, especially VAT, in normal weight young adults [12-14]. Recent advancements have allowed VAT to be accurately measured using Dual Energy X-Ray Absorptiometry (DXA) [15], making quantification of VAT more feasible.

Increased fitness usually through exercise is associated with improved metabolic outcomes and reduction of regional body fat [16-19], including VAT. Additionally, being fit during young adulthood is associated with reduced risk of developing prediabetes/diabetes in middle age [20-22]. However, in most cases these studies have used surrogate measures for fitness (submax tests or questionnaires), regional fat (waist circumference) and insulin sensitivity (HOMA-IR) [20-22]. Thus the association between fitness and fatness remains unknown in a normal weight population of young adults. Given the established relationship between VAT and cardiometabolic risk, reduced VAT mass in individuals with higher fitness levels may provide important information how higher fitness in young adulthood reduces the risk of future complications in glucose metabolism in sedentary young adults with the same BMI. The purpose of this 
pilot study was to characterize differences in insulin sensitivity and regional fat, including VAT, based on fitness level $\left(\mathrm{VO}_{2}\right.$ peak) in normal weight young adults matched on age, sex and BMI. We hypothesize that increased $\mathrm{VO}_{2}$ peak (fitness) will be associated with reduced VAT and improved insulin sensitivity. By using gold standard techniques to assess our outcome variables we may be able to identify key factors that influence future risk of dysfunctional glucose metabolism.

\section{Methods}

Thirty-eight young adults (age 19-31 years) were recruited from the University of Minnesota-Twin Cities campus and surrounding metropolitan area. Participants were screened for Fitness level was assessed by indirect calorimetry (Medgraphics CPX-D metabolic cart, Medical Graphics Corporation, St. Paul, MN) using a maximal oxygen consumption $\left(\mathrm{VO}_{2}\right.$ peak) using a treadmill test to exhaustion. Based on current physical activity status using self-report from the short-form International Physical Activity Questionnaire (IPAQ), a validated physical activity questionnaire [23], $\mathrm{VO}_{2}$ peak was measured in the sedentary ( $<30$ min exercise/ week) group using the Bruce protocol and physically active participants ( $>5$ days $>45 \mathrm{~min} /$ day) completed a progressive incline protocol at a set speed with increasing $2.5 \%$ incline every 2 minutes. Two different protocols were used to ensure a valid maximal test was completed within 12 minutes for current level of physical activity. Following fitness testing, participants were grouped into high (HF) and low fitness (LF) group based on being above or below the median for $\mathrm{VO}_{2}$ peak. Sixteen participants from each group ( 9 males/7 females) were matched on age ( \pm 2 years), gender and BMI $\left( \pm 2 \mathrm{~kg} / \mathrm{m}^{2}\right.$ ) and having a difference in $\mathrm{VO}_{2}$ peak $\geq 8 \mathrm{ml} / \mathrm{kg} / \mathrm{min}$. Six individuals $(2 \mathrm{M} / 4 \mathrm{~F})$ could not be matched and were excluded from the final analyses. The average BMI for the whole sample was $22.2 \mathrm{~kg} / \mathrm{m}^{2}$ (range 19.1-24.9 kg/ $\mathrm{m}^{2}$ ). Participants were excluded if they were currently using medications that affected lipid levels or glucose metabolism or consumed greater than $45 \%$ dietary fat as measured by a screening questionnaire [24], were pregnant, or experienced a recent weight change ( $>5$ pounds within the last 3 months). At least one week after fitness testing, participants completed a full body composition scan using a Lunar Prodigy (GE Healthcare, Madison, WI) Dual Energy X-Ray Absorptiometry (DXA) and had insulin sensitivity measured by hyperinsulinemic-euglycemic clamp on separate days. Women were studied in the follicular phase of their menstrual cycle. This protocol was approved by the University of Minnesota Institutional Review Board and consent was obtained from each participant.

\section{Body composition and maximal oxygen consumption}

Total body composition was measured using DXA (Lunar Prodigy, General Electric Medical Systems, Madison, WI, USA) and analyzed using its encore ${ }^{\mathrm{TM}}$ software (platform version 13.6 rev. 2). Participants were scanned using standard imaging and positioning protocols while fasted and hydrated. Subcutaneous fat (total, android and gynoid) and visceral fat were estimated using a method described previously for adults [15]. The android region was defined with a caudal limit placed at the top of the iliac crest and its height set to $20 \%$ of the distance from the top of the iliac crest to the base of the skull [25]. The gynoid region is located mid-pelvis to mid-thigh; the upper limit was set below the iliac crest a distance 1.5 times the height of the android region. The lower limit was set a distance of 2 times the height of the android region [25]. Trunk fat is fat within the region defined from the base of the mandible and includes the chest, abdomen and pelvic triangle, but excludes the arms by a boundary bisects the shoulder joint. All scans were reviewed for accurate placement of the android box by the same technician.

Maximal Oxygen Consumption $\left(\mathrm{VO}_{2}\right.$ peak) was determined with a graded treadmill test until exhaustion. Expired oxygen and carbon dioxide concentrations and volumes were collected and analyzed using a Med Graphics CPX-D metabolic cart (MedGraphics CPX-D metabolic cart, Medical Graphics Corporation, St. Paul, MN).

\section{Insulin sensitivity and measurement of blood markers}

Insulin sensitivity was measured by the hyperinsulinemiceuglycemic clamp as previously described [26]. Insulin was infused at a constant rate of $10.4 \mathrm{pmol} / \mathrm{L} / \mathrm{kg} / \mathrm{min}$ for 3 hours, and glucose was infused at a variable rate to maintain euglycemia. Insulin sensitivity (M) was expressed as the glucose infusion rate (mg/kg (FFM)/min of glucose) during the last 40 minutes of the clamp, with adjustment for fat free mass (M/FFM). Fasting blood samples were collected for glucose, and lipid levels including triglycerides, High-Density Lipoprotein Cholesterol (HCL-C), and Low-Density Lipoprotein Cholesterol (LDL-C). All assays were conducted with standard procedures at the Fairview Diagnostic Laboratories, Fairview-University Medical Center (Minneapolis, MN), Centers for Disease Control and Prevention-certified laboratory.

\section{Statistical analysis}

Normality of the data was evaluated using the AndersonDarling test. A t-test was used to compare demographic, blood markers and insulin sensitivity between the high and low fitness groups with males and females combined (Two groups). Because of differences in fat accumulation between sexes, we stratified the HF and LF groups by sex (4 groups) for the body composition analyses. An analysis of variance (ANOVA) was used to compare total and regional body composition measurements and insulin sensitivity between HF and LF males and females (4 groups). A post-hoc analysis using Tukey honest significant difference compared the HF and LF groups stratified by sex. T-tests compared the average difference between male and female groups for a sex main effect.

\section{Results}

Table 1 presents the demographic and clinical data by fitness level (sexes combined). The HF and LF groups were matched by age, gender, and BMI. The HF group had significantly lower percent body fat $(\% \mathrm{BF})$ and higher $\mathrm{VO}_{2}$ peak than the LF group $(-6.8 \% P=0.01,+9.5 \mathrm{ml} / \mathrm{kg} / \mathrm{min} P<0.001)$. The HF group also had higher insulin sensitivity $(+3.5 \mathrm{mg} / \mathrm{kg}(\mathrm{FFM}) / \mathrm{min} P=0.002)$, as measured by the hyperinsulinemic-euglycemic clamp, than the LF group. 
Table 1: Baseline characteristics of trained and sedentary young adults mean $(\underline{ \pm s d})$.

\begin{tabular}{|c|c|c|c|}
\hline & Trained & Sedentary & $p$-value \\
\hline $\mathrm{n}$ & 21(10 males, 11 females) & $18(9$ males, 9 females $)$ & \\
\hline Age (yr.) & $23(4)$ & $22(2)$ & 0.08 \\
\hline Height $(\mathrm{cm})$ & $171.4(10.3)$ & $167.3(7.6)$ & 0.25 \\
\hline Weight (kg) & $66.1(11.3)$ & $62.0(10.2)$ & 0.20 \\
\hline BMI $\left(\mathrm{kg} / \mathrm{m}^{2}\right)$ & $22.4(2.1)$ & $22.0(2.7)$ & 0.46 \\
\hline $\mathrm{VO}_{2} \max (\mathrm{ml} / \mathrm{kg} / \mathrm{min})$ & $48.5 *(7.0)$ & $39.0(5.3)$ & $<0.001$ \\
\hline Free Fatty Acids (mmol/L) & $0.3(0.2)$ & $0.4(0.2)$ & 0.15 \\
\hline Triglycerides (mmol/L) & $0.96(0.3)$ & $0.79(0.2)$ & 0.11 \\
\hline HDL-C (mmol/L) & $1.5(0.3)$ & $1.4(0.2)$ & 0.41 \\
\hline LDL-C (mmol/L) & $2.5(0.7)$ & $2.3(0.6)$ & 0.51 \\
\hline Fasting Glucose (mmol/L) & $4.6(0.6)$ & $4.6(0.5)$ & 0.64 \\
\hline Insulin (pmol/L) & $15.6^{*}(18.0)$ & $30.0(20.0)$ & 0.04 \\
\hline $\mathrm{M}_{\mathrm{ffm}}\left(\mathrm{mg} / \mathrm{kg}_{(\mathrm{ffm})} / \mathrm{min}\right.$ & $12.4^{*}(2.8)$ & $8.9(2.5)$ & 0.002 \\
\hline
\end{tabular}

$p$-value threshold $(<0.05)$

HDL-C = High-Density Lipoprotein Cholesterol, LDL-C = Low-Density Lipoprotein Cholesterol

$\mathrm{Mlbm}=$ Insulin Sensitivity, $\mathrm{M}$ corrected for fat free mass

Table 2 presents the body composition characteristics and insulin sensitivity comparisons for fitness level and sex. Higher fitness was associated with significantly lower $\% \mathrm{BF}$, trunk fat, android fat and subcutaneous abdominal fat $(P<0.05$ for all). Additionally higher fitness was associated with higher total lean mass, leg lean mass and insulin sensitivity in males $(P<0.05$ for all). In females, higher fitness was associated with lower \%BF and lower leg fat. However, there were no other differences in regional fat or lean mass as well as insulin sensitivity between $\mathrm{HF}$ and LF females $(-6.7 \%, P=0.001 ;-2.7 \mathrm{~kg}, P<0.001 ; 2.5 \mathrm{mg} /$ $\mathrm{kg}(\mathrm{FFM}) / \mathrm{min}, P=0.40)$. Interestingly in females, there was no difference in total lean mass, trunk lean mass or leg lean mass $(P=0.59, P=0.17, P=0.99)$. In both males and females there was no differences in VAT $(P>0.05$ for all $)$.

\section{Discussion}

The purpose of this study was to compare total and regional body composition and insulin sensitivity between HF and LF young adults with normal BMI. Interestingly, total fat was not different between HF and LF males and females; however, \%BF was significantly lower in HF males and females compared to LF males and females. There was an effect of sex on total and

Table 2: Comparison of participants by activity level and gender mean $( \pm \mathrm{SD})$.

\begin{tabular}{|c|c|c|c|c|c|}
\hline & \multicolumn{2}{|c|}{ Males $(n=18)$} & \multicolumn{2}{|c|}{ Females $(n=14)$} & \multirow{2}{*}{$\begin{array}{l}\text { Mean pair } \\
\text { differences } \\
P \text {-value }\end{array}$} \\
\hline & Low Fitness $(n=9)$ & High Fitness ( $n=9)$ & Low Fitness $(n=7)$ & High Fitness $(n=7)$ & \\
\hline $\mathrm{VO}_{2}$ peak $(\mathrm{ml} / \mathrm{kg} / \mathrm{min})$ & $41.0^{\mathrm{A}}(4.9)$ & $51.9^{\mathrm{B}}(6.2)$ & $36.4^{\mathrm{A}}(4.7)$ & $44.8^{\mathrm{C}}(4.5)$ & 0.39 \\
\hline BMI $\left(\mathrm{kg} / \mathrm{m}^{2}\right)$ & $22.5^{A}(3.0)$ & $23.0^{A}(1.8)$ & $21.6^{A}(2.4)$ & $21.7^{A}(2.3)$ & 0.44 \\
\hline Percent Fat (kg) & $24.0^{A}(5.5)$ & $16.8^{\mathrm{B}}(2.3)$ & $32.5^{c}(4.7)$ & $25.8^{A}(4.9)$ & 0.99 \\
\hline Total Fat (kg) & $15.8^{\mathrm{AB}}(5.7)$ & $11.8^{\mathrm{B}}(1.9)$ & $17.8^{A}(3.8)$ & $14.6^{\mathrm{AB}}(4.2)$ & 0.99 \\
\hline Total Lean (kg) & $48.0^{A}(5.8)$ & $59.6^{\mathrm{B}}(7.8)$ & $37.6^{c}(3.6)$ & $41.5^{A C}(3.2)$ & 0.06 \\
\hline Trunk Fat (kg) & $7.9^{A}(3.3)$ & $5.1^{\mathrm{B}}(0.8)$ & $7.5^{A}(1.6)$ & $6.6^{\mathrm{AB}}(2.7)$ & 0.25 \\
\hline Trunk Lean (kg) & $21.9^{A}(2.8)$ & $28.1^{\mathrm{B}}(3.7)$ & $17.2^{\mathrm{C}}(1.6)$ & $20.3^{A C}(1.5)$ & 0.13 \\
\hline Legs Fat (kg) & $5.3^{A}(2.0)$ & $4.9^{A}(0.9)$ & $8.0^{\mathrm{B}}(1.9)$ & $5.3^{A}(1.3)$ & 0.09 \\
\hline Legs Lean (kg) & $17.1^{A}(2.3)$ & $20.6^{\mathrm{B}}(2.8)$ & $14.0^{\mathrm{C}}(1.6)$ & $14.1^{A C}(1.8)$ & 0.04 \\
\hline Android Fat (kg) & $1.1^{A}(0.6)$ & $0.6^{\mathrm{B}}(0.2)$ & $1.0^{\mathrm{AB}}(0.2)$ & $0.9^{\mathrm{AB}}(0.5)$ & 0.18 \\
\hline Gynoid Fat (kg) & $2.6^{A}(1.1)$ & $2.0^{\mathrm{A}}(0.5)$ & $3.6^{B}(0.9)$ & $2.8^{\mathrm{AB}}(0.9)$ & 0.51 \\
\hline $\mathrm{A} / \mathrm{G}$ ratio & $0.42^{\mathrm{A}}(0.1)$ & $0.28^{\mathrm{B}}(0.1)$ & $0.27^{\mathrm{B}}(0.1)$ & $0.31^{\mathrm{AB}}(0.1)$ & 0.04 \\
\hline Subq fat (kg) & $0.9^{A}(0.5)$ & $0.5^{\mathrm{B}}(0.2)$ & $1.0^{\mathrm{A}}(0.2)$ & $0.7^{\mathrm{AB}}(0.5)$ & 0.23 \\
\hline Visceral Fat (kg) & $0.2^{A}(0.2)$ & $0.1^{A}(0.1)$ & $0.1^{A}(0.1)$ & $0.2^{A}(0.1)$ & 0.19 \\
\hline $\begin{array}{l}\text { Insulin Sensitivity (mg/ } \\
\operatorname{kg}_{\text {FFM }} / \text { min) }\end{array}$ & $8.2^{\mathrm{A}}(1.8)$ & $13.8^{\mathrm{B}}(3.7)$ & $9.1^{A}(3.0)$ & $11.6^{\mathrm{AB}}(2.7)$ & 0.45 \\
\hline
\end{tabular}

Groups that do not share a letter within the same row are significantly different at $P=0.05$ adjusted for multiple comparisons and small sample size. $\mathrm{A} / \mathrm{G}$ ratio is ratio of android fat over gynoid fat

Subq is the subcutaneous android (abdominal) fat depot

Mean pair differences compares the average difference between each pair between males and females (ex. Average difference for percent fat between male and female pairs) 
regional composition differences. HF males had higher total lean mass and lower regional fat as most locations compared to LF males. In contrast HF females only had significantly lower leg fat compared to LF females. Contrary to our hypotheses there was no significant difference in VAT mass between fitness levels. Sex also influenced insulin sensitivity differences between HF and LF individuals. HF males were more insulin sensitive than LF males but the same was not observed for females. These results suggest that higher fitness influences the proportion of lean and fat mass and those regional differences may be sex specific.

Overall, these results in a younger population are consistent with previous research in older and heavier adults [14-18]. However, this study observed differences between males and females between regional fat and lean mass between young adults with different fitness levels. These differences could be important in future interventional studies. While crosssectional in design, these results suggest that higher fitness has a sex specific effect on regional fat mass and lean mass. These differences may explain why higher insulin sensitivity was only observed in males with higher fitness. The lack of difference in VAT mass may be explained by recently identified \%BF thresholds for VAT accumulation in males and females [27,28]; both males and females were below or near these thresholds and would not have started accumulating VAT. However, LF males and females had significantly higher $\% \mathrm{BF}$, longitudinal studies are needed to ascertain if this puts LF individuals at greater risk for future cardiometabolic complications (since they are closer to the VAT accumulation threshold). Interestingly, other depots have been associated with changes in insulin sensitivity [29] and may explain the sex differences in insulin sensitivity between HF and LF males and females. Training intensity or training volume differences between HF males and females may explain the regional lean mass differences between males and females. However, this study suggests that improved fitness results in sexspecific differences in regional body composition.

Generally, males store more fat in the abdominal region and females store fat in the lower body. This could explain why HF males and females had difference patterns of regional fat compared to their LF groups. These results suggest that in males, higher fitness (usually associated with higher physical activity levels) is associated with lower \%BF, lower regional fat and higher insulin sensitivity. In females however, higher fitness was associated with lower $\% \mathrm{BF}$ and lower leg fat but no difference in insulin sensitivity or lean mass differences. A previous study observed no change in leg fat mass following a six-month intervention using resistance training [29], suggesting that different training modalities may result in differential changes in regional body composition. While fitness level was not associated with any difference in standard clinical blood markers (ex. lipids, cholesterol, etc.) insulin was significantly higher in the LF group (Table 1: males and females combined). Additionally it has been observed that higher baseline fitness levels are associated with a lower incidence of future cardiovascular disease and prediabetes/diabetes [20-22]. Future studies should examine the role of $\% \mathrm{BF}$ over time as it may be an influencing factor.
The strengths of this study were the use of gold standard measurements for assessing body composition, fitness level and insulin sensitivity [30]. The primary weakness was the small sample size, which may have limited the statistical power to detect differences between groups, however this was a pilot study aimed at identifying regional fat and lean mass differences between HF and LF young adults with normal BMI. Additionally, the small sample size limited our ability to control of other factors that affect body composition (ex. occupation etc.). These results have identified significant \%BF differences in males and females with normal BMI that may influence future cardiometabolic risk. Differences in \%BF may explain the metabolically unhealthy normal-weight individuals as well as those with obesity who are metabolically healthy [31].

\section{Conclusion}

This study highlights that even in young adults classified with normal BMI, having a higher fitness level is associated with a more favorable body composition. Sex influences regional fat differences between $\mathrm{HF}$ and LF males and females, but does not have any effect on VAT mass. Higher \%BF during young adulthood, observed in both LF males and females, may influence future metabolic risk and VAT accumulation but has no association with cardiovascular risk factors in young adults with normal BMI.

\section{Acknowledgements}

LC conceived and carried out the experiments, TB and DD analyzed data. All authors contributed to data interpretation and all authors were involved in writing the paper and had final approval of the submitted and published versions. Grant support was by National Institutes of Health (5K12RR023247-02), Minnesota Medical Foundation, Pennock Family Foundation, University of Minnesota CTSA: NIH UL1TR000114, Metabolic Studies Core of the Minnesota Obesity Center: Supported by NIH DK50456, Mayo CTSA: NIH UL1 TR000135.

\section{References}

1. Gómez-Ambrosi J, Silva C, Galofré JC, Escalada J, Santos S, Gil MJ, et al. Body adiposity and type 2 diabetes: increased risk with a high body fat percentage even having a normal BMI. Obesity (Silver Spring). 2011; 19(7):1439-1444. doi: 10.1038/oby.2011.36.

2. Gómez-Ambrosi J, Silva C, Galofré JC, Escalada J, Santos S, Millán D, et al. Body mass index classification misses subjects with increased cardiometabolic risk factors related to elevated adiposity. Int J Obes (Lond). 2012; 36(2):286-294. doi: 10.1038/ijo.2011.100.

3. McLaughlin T, Lamendola C, Liu A, Abbasi F. Preferential fat deposition in subcutaneous versus visceral depots is associated with insulin sensitivity. J Clin Endocrinol Metab. 2011; 96(11):E1756-60. doi: 10.1210/jc.2011-0615.

4. Porter SA, Massaro J M, Hoffmann U, Vasan R S, O’Donnel, CJ, Fox CS. Abdominal subcutaneous adipose tissue: a protective fat depot? Diabetes Care. 2009; 32(6):1068-1075. doi: 10.2337/dc08-2280.

5. Wiklund P, Toss F, Weinehall L, Hallmans G, Franks PW, Nordström A, et al. Abdominal and gynoid fat mass are associated with cardiovascular risk factors in men and women. J Clin Endocrinol Metab. 2008 ; 93(11):4360-4366. doi: 10.1210/jc.2008-0804. 
6. Després JP, Lemieux I, Prud'homme D. Treatment of obesity: need to focus on high risk abdominally obese patients. BMJ. 2001; 322(7288):716-720.

7. Neeland IJ, Turer AT, Ayers CR, Powell-Wiley TM, Vega, GL FarzanehFar R, et al. Dysfunctional Adiposity and the Risk of Prediabetes and Type 2 diabetes in Obese Adults. JAMA. 2012; 308(11):1150-1159.

8. Rothney MP, Catapano AL, Xia J, Wacker WK, Tidone C, Grigore L, et al. Abdominal visceral fat measurement using dual-energy X-ray: Association with cardiometabolic risk factors. Obesity (Silver Spring). 2013; 21(9):1798-1802. doi: 10.1002/oby.20223.

9. Taylor RW, Grant AM, Williams SM, Goulding A. Sex Differences in Regional Body Fat Distribution From Pre-to Postpuberty. Obesity (Silver Spring). 2010; 18(7):1410-1416. doi: 10.1038/oby.2009.399.

10. Yan S, Zhao X, Shen X, Yang L, Yuan X, Huang L, et al. Abnormal Regional Body Fat Distribution Also Exists in Non-Obese Subjects with High Blood Pressure. Clin Exp Hypertens. 2013; 35(7):528-533. doi: 10.3109/10641963.2013.764886.

11. Patel $P$ \& Abate N. Body fat distribution and insulin resistance Nutrients. 2013; 5(6):2019-2027. doi: 10.3390/nu5062019.

12. Kohrt WM, Malley MT, Dalsky GP, Holloszy JO. Body composition of healthy sedentary and trained, young and older men and women. Med Sci Sports Exerc. 1992; 24(7):832-837.

13. Mundi MS, Karpyak MV, Koutsari C, Votruba SB, O’Brien PC, Jensen MD Body fat distribution, adipocyte size, and metabolic characteristics of nondiabetic adults. J Clin Endocrinol Metab. 2010; 95(1):67-73. doi:10.1210/jc.2009-1353.

14. Hermsdorff HH, Zulet MÁ, Puchau B, Martínez JA. Central adiposity rather than total adiposity measurements are specifically involved in the inflammatory status from healthy young adults. Inflammation. 2011; 34(3):161-170. doi: 10.1007/s10753-010-9219-y.

15. Kaul S, Rothney MP, Peters DM, Wacker WK, Davis CE, Shapiro MD, et al. Dual-energy X-ray absorptiometry for quantification of visceral fat. Obesity (Silver Spring). 2012; 20(6):1313-1318. doi: 10.1038/ oby.2011.393.

16. Wong SL, Katzmarzyk PT, Nichaman MZ, Church TS, Blair SN, Ross R Cardiorespiratory Fitness is Associated with Lower Abdominal Fat Independent of Body Mass Index. Med Sci Sports Exerc 36(2):286-291.

17. Lee S, Kuk JL, Davidson LE, Hudson R, Kilpatrick K, Graham TE, et al. Exercise without weight loss is an effective strategy for obesity reduction in obese individuals with Type 2 diabetes. J Appl Physiol (1985). 2005; 99(3):1220-1225.

18. Perez-Gomez J, Vicente-Rodríguez G, Ara Royo I, Martínez-Redondo D, Puzo Foncillas J, Moreno LA, et al. Effect of endurance and resistance training on regional fat mass and lipid profile. Nutr Hosp. 2013; 28(2):340-346. doi: 10.3305/nh.2013.28.2.6200.

19. Perseghin G, Price TB, Petersen KF, Michael Roden, Gary W. Cline, Karynn Gerow, et al. Increased glucose transport-phosphorylation and muscle glycogen synthesis after exercise training in insulin resistant subjects. New England Journal of Medicine 1996; 335(18):1357-1362. DOI: 10.1056/NEJM199610313351804.

20. Blair SN, Kampert JB, Kohl HW, Barlow CE, Macera CA, Paffenbarger RS $\mathrm{Jr}$, et al. Influences of cardiorespiratory fitness and other precursors on cardiovascular disease and all-cause mortality in men and women. JAMA. 1996; 276(3):205-210.

21. Carnethon MR, Gidding SS, Nehgme R, Sidney S, Jacobs DR Jr, Liu K. Cardiorespiratory fitness in young adulthood and the development of cardiovascular disease risk factors. JAMA. 2003; 290(23):3092-3100.

22. Carnethon MR, Sternfeld B, Schreiner PJ, Jacobs DR Jr, Lewis CE, Liu $\mathrm{K}$, et al. Association of 20-year changes in cardiorespiratory fitness with incident type 2 diabetes: the coronary artery risk development in young adults (CARDIA) fitness study. Diabetes Care. 2009; 32(7):1284-1288. doi: 10.2337/dc08-1971.

23. Craig CL, Marshall AL, Sjöström M, Bauman AE, Booth ML, Ainsworth $\mathrm{BE}$, et al. International physical activity questionnaire: 12-country reliability and validity. Med Sci Sports Exerc 2003; 35:1381-1395.

24. Block G, Gillespie C, Rosenbaum EH, Jenson C. A rapid food screener to assess fat and fruit and vegetable intake. Am J Prev Med 2000; 18(4):284-288.

25. Stults-Kolehmainen MA, Stanforth PR, Bartholomew JB, Lu T, Abolt CJ, Sinha R. DXA estimates of fat in abdominal, trunk and hip regions varies by ethnicity in men. Nutr Diabetes. 2013; 3:e64. doi: 10.1038/ nutd.2013.5.

26. Chow LS, Seaquist ER, Eberly LE, Mashek MT, Schimke JM, Nair $\mathrm{KS}$, et al. Acute Free Fatty Acid Elevation Eliminates Endurance Training Effect on Insulin Sensitivity. J Clin Endocrinol Metab. 2012; 97(8):2890-2897. doi: 10.1210/jc.2012-1515.

27. Bosch TA, Burruss TP, Weir NL, Fielding KA, Engel BE, Weston TD, et al. Abdominal Body Composition Differences in NFL Football Players. J Strength Cond Res. 2014; 28(12):3313-9. doi:10.1519/ JSC.0000000000000650.

28. Bosch TA, Steinberger J, Sinaiko AR, Moran A, Jacobs DR Jr, Kelly AS, et al. Identification of sex-specific thresholds for accumulation of visceral adipose tissue in adults. Obesity (Silver Spring). 2015 Feb;23(2):37582. doi: 10.1002 /oby.20961.

29. Goodpaster BH, Thaete FL, Simoneau JA, Kelley DE. Subcutaneous abdominal fat and thigh muscle composition predict insulin sensitivity independently of visceral fat. Diabetes 1997; 46(10): 1579-1585.

30. Nindl BC, Harman EA, Marx JO, Gotshalk LA, Frykman PN, Lammi E, et al. Regional body composition changes in women after 6 months of periodized physical training. J Appl Physiol 2000; 88:2251-2259.

31. Karelis AD, Faraj M, Bastard JP, St-Pierre DH, Brochu M, Prud'homme $D$, et al. The metabolically healthy but obese individual presents a favorable inflammation profile. J Clin Endocrinol Metab. 2005; $90(7): 4145-4150$ 